\title{
Acknowledgement of manuscript reviewers 2019
}

\section{Victoria G. Vivilaki ${ }^{1}$}

\section{CONTRIBUTING REVIEWERS}

The editors of European Journal of Midwifery would like to thank all our reviewers who have contributed to the journal in Volume 3 (2019).

\section{Alé, Franck}

France

Alipour, zahra

Iran

Antonakou, Angeliki

United Kingdom

Baird, Kathleen

Australia

\section{Betron, Myra}

United States

Bonilla, Hilda

Chile

Curtis, Kath

United States

Darra, Susanne

United Kingdom

Dey, Teesta

United Kingdom

Dieb, Amira

Egypt

Durain, Dawn

United States
Egenberg, Signe

Norway

Escuriet, Ramón

Spain

Forbes, Faye

Australia

Fox, Deborah

Australia

Grum, Teklit

Ethiopia

Harris, Melissa

Australia

Hisano, Michi

Japan

Hunter, Billie

United Kingdom

King, Rosemary

Australia

Lagana, Antonio

Italy

Lambert, Jaki

United Kingdom

\section{AFFILIATION}

1 Department of Midwifery, University of West Attica, Athens, Greece

\section{CORRESPONDENGE TO}

Victoria Vivilaki. Department of Midwifery,

University of West Attica, Athens, Greece.

E-mail: v.vivilaki@gmail.com
Mackenzie, Ian

United Kingdom

Mas, Masresha L.

Ethiopia

Mc Carthy, Jane

Ireland

Meneses-Rentería, Alba

Mexico

Mitchell, Creina

Australia

Mivšek, Polona

Slovenia

Münstedt, Karsten

Germany

Ny, Pernilla

Sweden

Oo, Win

Malaysia

Prosen, Mirko

Slovenia

Sandwell, Rachel

Canada 
Sharek, Danika

Ireland

Teklesilasie, Wondwosen

Ethiopia

\section{Tura, Halkeno}

United States
Vermeulen, Joeri

Belgium

Werner, Anette

Denmark

Whitburn, Laura

Australia
Wier, Jacqueline

United Kingdom

\section{Yurdakul, Mine}

Turkey 